\title{
Transvaginal Resection of a Rectal Gastrointestinal Stromal Tumor: A Case Report
}

\author{
Marta M. Ferreira ${ }^{\mathrm{a}, \mathrm{b}}$, Narcisa Guimaraes ${ }^{\mathrm{a}}$, Ines Bolais Monica ${ }^{\mathrm{a}}$, Simone Oliveira ${ }^{\mathrm{a}}$, \\ Daniela Pato Pais ${ }^{\mathrm{a}}$, Sara Andrade ${ }^{\mathrm{a}}$, Nuno Azenha ${ }^{\mathrm{a}}$, Raquel Dias ${ }^{\mathrm{a}}$, \\ Isabel Borges ${ }^{\mathrm{a}}$, Jose Valente Cecilio ${ }^{\mathrm{a}}$
}

\begin{abstract}
Gastrointestinal stromal tumors (GISTs) are a type of mesenchymal tumors. They are rare in rectum. This location is a surgical challenge due to the high morbidity after aggressive surgeries. We present the case of an 81-year-old female with a high-grade GIST treated with transvaginal excision and adjunctive therapy with imatinib. The postoperative period was uneventful without anal dysfunction. Within the types of surgical treatment available, transvaginal excision is an acceptable procedure in lesions located in the rectovaginal region.
\end{abstract}

Keywords: Gastrointestinal stromal tumor; Rectum; Transvaginal resection

\section{Introduction}

Gastrointestinal stromal tumors (GISTs) are relatively uncommon in rectum $(5 \%)$. They are mainly found in stomach $(60 \%)$ and small intestine (30\%) [1], and constitute $0.1 \%$ of all tumors with rectal origin [2].

The treatment of choice for resectable rectal GIST is the complete resection, which is the most effective treatment, with the lowest risk of recurrence [2,3]. The aim is to do the lesion excision (R0 resection), without violating the lesion itself. In the low rectum, there must be taken account the proximity of the anal sphincters, with risks of complications like anal incontinence or sexual or urological dysfunction in more extensive resections [4]. GIST.

We report a case of an 81-year-old female with a rectal

Manuscript submitted November 1, 2020, accepted November 17, 2020

Published online December 30, 2020

aDepartment of Surgery, Hospital Distrital da Figueira da Foz, Rua do Hospital 3094-001, Figueira da Foz, Portugal

${ }^{b}$ Corresponding Author: Marta M. Ferreira, Department of Surgery, Hospital Distrital da Figueira da Foz, Rua do Hospital 3094-001, Figueira da Foz, Portugal. Email: martasmferreira@gmail.com

doi: https://doi.org/10.14740/jmc3615

\section{Case Report}

An 81-year-old female was brought to the Emergency Department of our hospital, due to recurrent episodes of rectal bleeding, without constitutional complaints. On rectal examination, it was identified an ulcerated rectal mass, on the anterior wall of the rectum. A colonoscopy was performed, which showed an ulcerated, hard and friable tumor located on the anterior surface of the distal rectum (Fig. 1a). The biopsy revealed a high-grade GIST. The patient was subjected to a computed tomography (CT) scan that showed a tumorous formation in the rectum, with no densification of the fat of the ischiorectal space or pelvic lymphadenopathy (Fig. 1b). The pelvic magnetic resonance imaging (MRI) scan results showed a $5.1 \times 3.1$ $\mathrm{cm}$ mass in the rectovaginal space with origin in the anterior wall of the rectum, $2.9 \mathrm{~cm}$ from the anal verge with presumably non-epithelial origin and no apparent local invasion (Fig. 1c).

The patient underwent transvaginal resection of the lesion. The wall closure was made by layers (rectal mucosa, vaginal musculature and vaginal mucosa) (Fig. 2a-h).

The immediate postoperative period was uneventful, and the patient was discharged from the hospital on the sixth postoperative day.

The results of histological examination revealed a rectal GIST, with high risk of biological behavior, 18 mitosis $/ 5 \mathrm{~mm}^{2}$, and positive for CD34, Dog-1 and c-Kit. The proliferative index (assessed with Ki67) was around 60-70\%.

On multidisciplinary consultation, the patient was proposed to adjunctive therapy with imatinib.

\section{Discussion}

GIST represents $80 \%$ of mesenchymal tumors. They are relatively rare, constituting $1-3 \%$ of all malignant gastrointestinal tumors [5]. The organs most often affected are the stomach $(60 \%)$, jejunum and ileum $(30 \%)$. The other locations are duodenum (5\%), colon and rectum $(5 \%)$ and esophagus and appendix $(<1 \%)$. Rarely, they can be found in extraluminous locations such as mesentery, omentum and retroperitoneum $(<5 \%)$ [6]. Rectal GISTs are more predominant in males and are more frequent between the fifth and seventh decade of life [7]. 

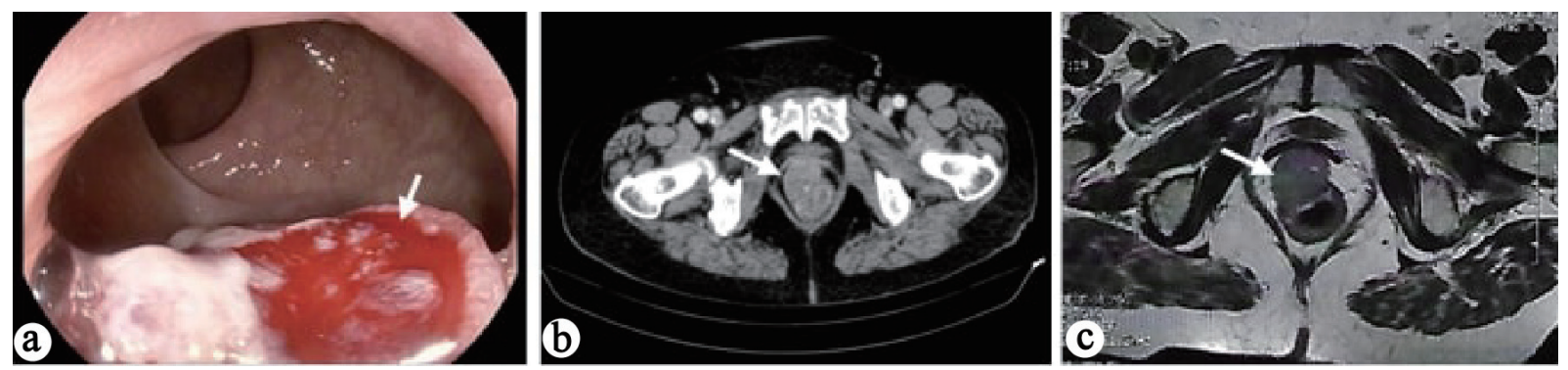

Figure 1. Complementary diagnostic tests. (a) Colonoscopy: ulcerated, hard and friable lesion (arrow), located on the anterior surface of the distal anal-rectal rectum. (b) CT scan: tumor formation (arrow) in the rectum, with no densification of the fat of the ischiorectal space or lymphadenopathy. (c) Pelvic MRI scan: mass (arrow) in the rectovaginal space of $5.1 \times 3.1 \mathrm{~cm}$ with origin in the anterior wall of the rectum about $2.9 \mathrm{~cm}$ from the anal canal with presumably non-epithelial origin and without apparent local invasion.

This type of tumor originates in interstitial cells of $\mathrm{Ca}$ jal, located in the myenteric plexus of the gastrointestinal wall, which act as gastrointestinal motility regulators [8]. Approximately $10-30 \%$ are clinically malignant; however, all of them have some potential degree of malignancy [9].

GISTs are associated with two mutations: the mutation of the $c$-kit proto-oncogene (responsible for the expression of the CD117 protein, a receptor for the tyrosine kinase growth factor) and the mutation of the platelet-derived growth factor receptor alpha (PDGFRA) gene. These mutations are present in $80-85 \%$ of patients $(75-80 \%$ c-kit and $10-15 \%$ PDGFRA). The GISTs that do not have these mutations are classified as "wild type", this one with better prognosis $[6,10]$.

Most patients are asymptomatic. In rectum, although rare, obstruction or hemorrhage may be present.
The diagnosis follows the principles of diagnosis of any rectal mass, using imaging exams (endoscopy, CT scan and pelvic MRI scan) and biopsy.

Usually, the GIST is endoscopically characterized as a bulging of the wall, with normal mucosa or eventual mucosal ulceration, with solid consistency [8].

The diagnosis includes CT scan with intravenous contrast, in order to identify distant metastatic disease. Locally they present as a well-circumscribed region of contrast, with heterogeneous enhancement, particularly if it is a large GIST with necro-hemorrhagic areas or degenerative components [11].

Pelvic MRI scan allows locoregional staging. The lesions are usually described as well-defined solid masses, and some of them may have central necrosis, presenting with a diffuse
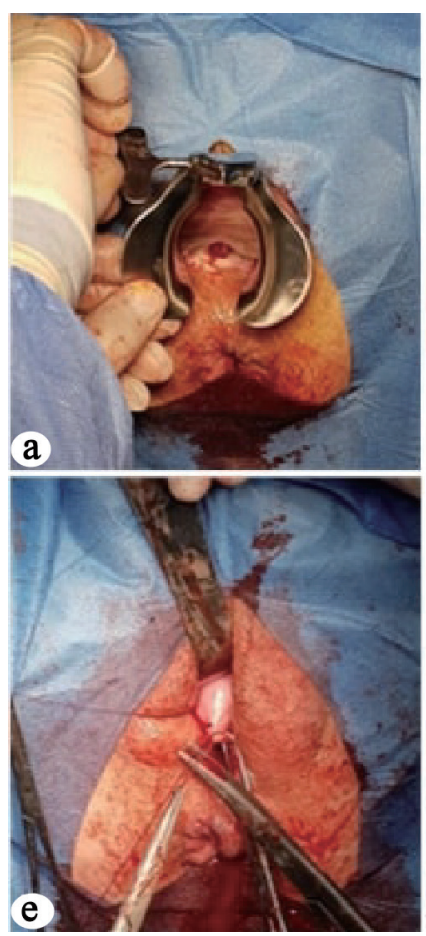
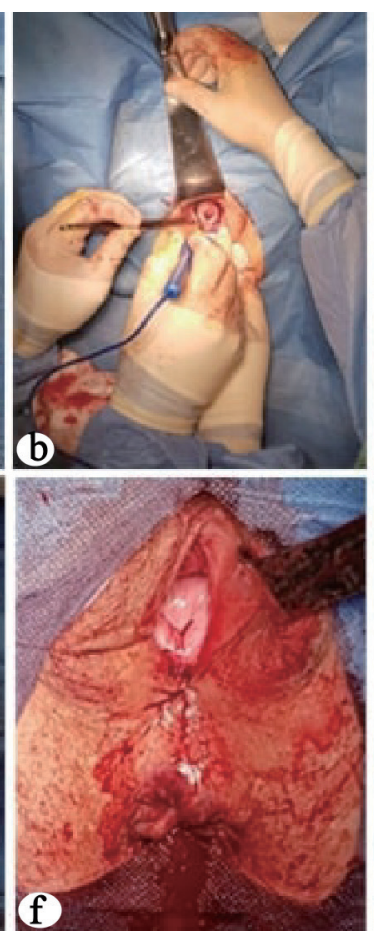
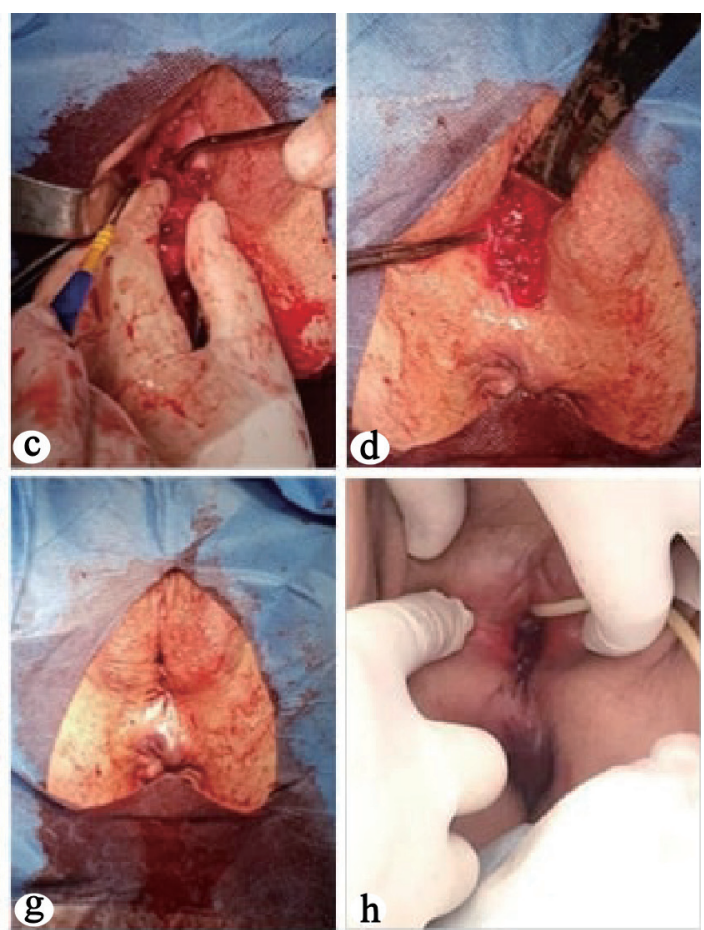

Figure 2. Surgical intervention: transvaginal resection. (a) Protrusion of the lesion through the posterior wall of the vagina. (b-d) Transvaginal excision of the lesion. (e-g) Defect closure by layers. (h) The first postoperative day. 
hyposignal at T1 and hypersignal at T2. Post-contrast gain is homogeneous in the solid region, forming a ring around central necrosis.

Signs of malignancy include ulceration, irregular margins and increased dimensions on follow-up exams [12].

Biopsy is particularly important in the characterization of the tumor, namely in the differential diagnosis with rectal adenocarcinoma (in the case of ulcerated GIST), whose management are different. The final diagnosis is established according to the specific histological and immunohistochemical characteristics (CD117, CD34, actin, desmin, S-100 and Ki67). Sometimes, the biopsy is not conclusive since it is a neoplasm originated in the deeper layers of the rectum, which, if not biopsied, there is no representative sample.

The standard treatment of non-metastatic GIST involves complete resection of the tumor, with free margins, without rupture of the capsule $[2,3]$. In the case of rectal GIST, the purpose of surgery, in addition to local tumor resection, is when possible, the sphincters preservation. The spread does not usually involve regional ganglion metastases, so regional lymphadenectomy is unnecessary [1].

There are several surgical techniques described, from conventional transanal resection (Parks), trans-sacral approach (Kraske), transanal endoscopic microsurgery (TEM), transanal minimally invasive surgery (TAMIS), laparoscopic and laparotomic surgery (anterior resection of the rectum, abdominalperineal amputation) [13-17]. The method of choice is decided according to the characteristics of the tumor (size, extent, location) and according to the surgeon's preferences.

The resection of tumors of the anterior wall of the rectum is technically more difficult. The transvaginal approach is a possible method for local tumor excision when located in the rectovaginal region $[18,19]$. It is an alternative to a low or ultralow anterior resection surgery, which has a higher risk of complications. Transvaginal resection is a valid approach due to the large volume of the vagina, which is enough to support the resection.

In our case, enucleation without fragmentation of the piece was not possible. However, the entire lesion was macroscopically resected, and that constitutes one of the most important factors for the prognosis.

In large tumors and in those that have a high risk of metastases, preoperative treatment with tyrosine kinase inhibitors such as imatinib is recommended to decrease tumor volume, improve intact resection with negative margins, sphincter preservation and increase disease-free survival [3, 13]. Several studies have shown that neoadjuvant treatment with imatinib improved R0 resection rates and decreased the risk of postoperative morbidity [20]. However, in the case of our patient, the tumor was symptomatic, requiring a primary surgical approach.

The worst prognostic factors for GIST are tumor size, mitotic activity ( $>5$ mitoses/50 high-power fields (a total area of $5 \mathrm{~mm}^{2}$ ), non-gastric location, male gender and pseudo-capsule rupture $[3,7]$.

Adjuvant therapy with tyrosine kinase inhibitor is strongly recommended in high-risk patients to reduce the risk of recurrence [20].

The biology of our patient's tumor, with a high risk of bio- logical behavior ( 18 mitosis $/ 5 \mathrm{~mm}^{2}$ and a proliferative index around $60-70 \%$ (Ki-67)), demanded adjuvant therapy [3].

Recurrence can develop 10 - 15 years after primary treatment, so clinical follow-up must be done over the long term [11].

\section{Conclusions}

Rectal GISTs are uncommon and the treatment involves local excision of the lesion, without regional lymphadenectomy. Transvaginal resection is an acceptable procedure in tumors located in the rectovaginal region, with protrusion in the posterior wall of the vagina. This method allows not only the excision of the tumor, but also prevents iatrogenic damage to the anal sphincters and their clinical consequences.

\section{Acknowledgments}

We appreciate the contributions of all surgeons.

\section{Financial Disclosure}

None to declare.

\section{Conflict of Interest}

None to declare.

\section{Informed Consent}

Consent was provided for the publication of images and necessary data.

\section{Author Contributions}

Marta Ferreira: corresponding and first author, conceptualization and study design, data acquisition, analysis and interpretation, article drafting and critical revision of the work for important intellectual content. Narcisa Guimaraes, Ines Bolais Monica, Simone Oliveira, Daniela Pato Pais, Sara Andrade: data analysis and interpretation, critical revision of the work for important intellectual content, and final approval of the manuscript to be published. Nuno Azenha, Raquel Dias, Isabel Borges, Jose Valente Cecilio: revising it critically for important intellectual content, final approval of the version to be published.

\section{Data Availability}

The data supporting the findings of this study are available from the corresponding author upon reasonable request. 


\section{References}

1. van der Zwan SM, DeMatteo RP. Gastrointestinal stromal tumor: 5 years later. Cancer. 2005;104(9):1781-1788.

2. Theodoropoulos DG. Gastrointestinal tumors of the colon and rectum. Clin Colon Rectal Surg. 2011;24(3):161-170.

3. Casali PG, Abecassis N, Aro HT, Bauer S, Biagini R, Bielack S, Bonvalot $\mathrm{S}$, et al. Gastrointestinal stromal tumours: ESMO-EURACAN Clinical Practice Guidelines for diagnosis, treatment and follow-up. Ann Oncol. 2018;29(Suppl 4):iv68-iv78.

4. Fiore M, Raut CP, Gronchi A. Are We Allowed to Limit Surgical Aggressiveness With Small Rectal Gastrointestinal Stromal Tumors? Ann Surg Oncol. 2017;24(5):11531156.

5. Cassier PA, Ducimetiere F, Lurkin A, Ranchere-Vince D, Scoazec JY, Bringuier PP, Decouvelaere AV, et al. A prospective epidemiological study of new incident GISTs during two consecutive years in Rhone Alpes region: incidence and molecular distribution of GIST in a European region. Br J Cancer. 2010;103(2):165-170.

6. Pisters PW, Patel SR. Gastrointestinal stromal tumors: current management. J Surg Oncol. 2010;102(5):530-538.

7. Miettinen M, Furlong M, Sarlomo-Rikala M, Burke A, Sobin LH, Lasota J. Gastrointestinal stromal tumors, intramural leiomyomas, and leiomyosarcomas in the rectum and anus: a clinicopathologic, immunohistochemical, and molecular genetic study of 144 cases. Am J Surg Pathol. 2001;25(9):1121-1133.

8. Sepe PS, Brugge WR. A guide for the diagnosis and management of gastrointestinal stromal cell tumors. Nat Rev Gastroenterol Hepatol. 2009;6(6):363-371.

9. Miettinen M, Lasota J. Gastrointestinal stromal tumors: review on morphology, molecular pathology, prognosis, and differential diagnosis. Arch Pathol Lab Med. 2006;130(10): 1466-1478.

10. Valsangkar N, Sehdev A, Misra S, Zimmers TA, O'Neil $\mathrm{BH}$, Koniaris LG. Current management of gastrointestinal stromal tumors: Surgery, current biomarkers, mutations, and therapy. Surgery. 2015;158(5):1149-1164.

11. Lee CM, Chen HC, Leung TK, Chen YY. Gastrointes- tinal stromal tumor: Computed tomographic features. World J Gastroenterol. 2004;10(16):2417-2418.

12. Nishida T. Asian consensus guidelines for gastrointestinal stromal tumor: what is the same and what is different from global guidelines. Transl Gastroenterol Hepatol. 2018;3:11.

13. Wilkinson MJ, Fitzgerald JE, Strauss DC, Hayes AJ, Thomas JM, Messiou C, Fisher C, et al. Surgical treatment of gastrointestinal stromal tumour of the rectum in the era of imatinib. Br J Surg. 2015;102(8):965-971.

14. Liu Q, Zhong G, Zhou W, Lin G. Initial application of transanal endoscopic microsurgery for high-risk lower rectal gastrointestinal stromal tumor after imatinib mesylate neoadjuvant chemotherapy: A case report. Medicine (Baltimore). 2017;96(29):e7538.

15. Kyo K, Azuma M, Okamoto K, Nishiyama M, Shimamura T, Maema A, Kanamaru H, et al. Neoadjuvant imatinib treatment and laparoscopic anus-preserving surgery for a large gastrointestinal stromal tumor of the rectum. World J Surg Oncol. 2016;14:68.

16. Nozawa $H$, Kanazawa $T$, Tanaka $T$, Takahashi M, Ishihara S, Sunami E, Kitayama J, et al. Laparoscopic resection of a gastrointestinal stromal tumor of the lower rectum in a patient with coronary artery disease following longterm neoadjuvant imatinib treatment and anticoagulation therapy. World J Surg Oncol. 2014;12:211.

17. Gervaz P, Huber O, Bucher P, Sappino P, Morel P. Transsacral (Kraske) approach for gastrointestinal stromal tumour of the lower rectum: old procedure for a new disease. Colorectal Dis. 2008;10(9):951-952.

18. Hellan M, Maker VK. Transvaginal excision of a large rectal stromal tumor: an alternative. Am J Surg. 2006;191(1):121-123.

19. Hara M, Takayama S, Arakawa A, Sato M, Nagasaki T, Takeyama $\mathrm{H}$. Transvaginal resection of a rectal gastrointestinal stromal tumor. Surg Today. 2012;42(9):909-912.

20. Joensuu H, Eriksson M, Sundby Hall K, Hartmann JT, Pink D, Schutte J, Ramadori G, et al. One vs three years of adjuvant imatinib for operable gastrointestinal stromal tumor: a randomized trial. JAMA. 2012;307(12):12651272. 